\title{
Cesàro Summable Sequence Spaces over the Non-Newtonian Complex Field
}

\author{
Ŭgur Kadak \\ Department of Mathematics, Faculty of Sciences and Arts, Bozok University, Turkey \\ Correspondence should be addressed to Uğur Kadak; ugurkadak@gmail.com
}

Received 12 September 2015; Accepted 27 October 2015

Academic Editor: Chin-Shang Li

Copyright (C) 2016 Uğur Kadak. This is an open access article distributed under the Creative Commons Attribution License, which permits unrestricted use, distribution, and reproduction in any medium, provided the original work is properly cited.

\begin{abstract}
The spaces $\omega_{0}^{p}, \omega^{p}$, and $\omega_{\infty}^{p}$ can be considered the sets of all sequences that are strongly summable to zero, strongly summable, and bounded, by the Cesàro method of order 1 with index $p$. Here we define the sets of sequences which are related to strong Cesàro summability over the non-Newtonian complex field by using two generator functions. Also we determine the $\beta$-duals of the new spaces and characterize matrix transformations on them into the sets of $*$-bounded, $*$-convergent, and $*$-null sequences of non-Newtonian complex numbers.
\end{abstract}

\section{Introduction}

The theory of sequence spaces is the fundamental of summability. Summability is a wide field of mathematics, mainly in analysis and functional analysis, and has many applications, for instance, in numerical analysis to speed up the rate of convergence, in operator theory, the theory of orthogonal series, and approximation theory. Also, the concepts of statistical convergence have been studied by various mathematicians. In recent years, generalizations of statistical convergence have appeared in the study of strong integral summability and the structure of ideals of bounded continuous functions on locally compact spaces. Many important sequence spaces arise in a natural way from different notions of summability, that is, ordinary, absolute, and strong summability. The first two cases may be considered as the domains of the matrices that define the respective methods; the situation, however, is different and more complicated in the case of strong summability. Many authors have extensively developed the theory of the matrix transformations between some sequence spaces; we refer the reader to [1-6].

As an alternative to the classical calculus, Grossman and Katz [7-9] introduced the non-Newtonian calculus consisting of the branches of geometric, quadratic, and harmonic calculus, and so forth. All these calculi can be described simultaneously within the framework of a general theory. They decided to use the adjective non-Newtonian to indicate any of calculi other than the classical calculus. Every property in classical calculus has an analogue in non-Newtonian calculus which is a methodology that allows one to have a different look at problems which can be investigated via calculus. In some cases, for example, for wage-rate (in dollars, euro, etc.) related problems, the use of bigeometric calculus which is a kind of non-Newtonian calculus is advocated instead of a traditional Newtonian one.

Many authors have extensively developed the notion of multiplicative calculus; see [10-12] for details. Also some authors have also worked on the classical sequence spaces and related topics by using non-Newtonian calculus [13-15]. Further Kadak [16] and Kadak et al. [17, 18] have matrix transformations between certain sequence spaces over the non-Newtonian complex field and have generalized RungeKutta method with respect to the non-Newtonian calculus.

The main focus of this work is to extend the strong Cesàro summable sequence spaces defined earlier to their generalized sequence spaces over the non-Newtonian complex field by using various generator functions, that is, exp and $q_{r}$ generators.

\section{Preliminaries, Background, and Notations}

Arithmetic is any system that satisfies the whole of the ordered field axioms whose domain is a subset of $\mathbb{R}$. There 
are infinitely many types of arithmetic, all of which are isomorphic, that is, structurally equivalent.

A generator is a one-to-one function whose domain is $\mathbb{R}$ and whose range is a subset $\mathbb{R}_{\alpha}$ of $\mathbb{R}$ where $\mathbb{R}_{\alpha}=\{\alpha(x)$ : $x \in \mathbb{R}\}$. Each generator generates exactly one arithmetic, and conversely each arithmetic is generated by exactly one generator. If $I(x)=x$ for all $x \in \mathbb{R}$, then $I$ is called identity function whose inverse is itself. In the special cases $\alpha=I$ and $\alpha=\exp , \alpha$ generates the classical and geometric arithmetic, respectively. By $\alpha$-arithmetic, we mean the arithmetic whose domain is $\mathbb{R}$ and whose operations are defined as follows. For $x, y \in \mathbb{R}_{\alpha}$ and any generator $\alpha$,

$$
\begin{gathered}
\alpha \text {-addition } x \dot{y}=\alpha\left\{\alpha^{-1}(x)+\alpha^{-1}(y)\right\} \\
\alpha \text {-subtraction } x-y=\alpha\left\{\alpha^{-1}(x)-\alpha^{-1}(y)\right\} \\
\alpha \text {-multiplication } x \dot{\times} y=\alpha\left\{\alpha^{-1}(x) \times \alpha^{-1}(y)\right\} \\
\alpha \text {-division } x \dot{y}=\alpha\left\{\alpha^{-1}(x) \div \alpha^{-1}(y)\right\} \\
\alpha \text {-order } x \dot{<} y \Longleftrightarrow \alpha^{-1}(x)<\alpha^{-1}(y) .
\end{gathered}
$$

As an example if we choose exp function from $\mathbb{R}$ to the set $\mathbb{R}_{\exp } \subseteq \mathbb{R}^{+}$,

$$
\begin{aligned}
\alpha: \mathbb{R} & \longrightarrow \mathbb{R}_{\exp } \\
x & \longmapsto y=\alpha(x)=e^{x},
\end{aligned}
$$

and $\alpha$-arithmetic turns out to be Geometric arithmetic:

$$
\begin{gathered}
\alpha \text {-addition } x \dot{y}=e^{\{\ln x+\ln y\}}=x \cdot y \\
\alpha \text {-subtraction } x \dot{-} y=e^{\{\ln x-\ln y\}}=x \div y \\
\alpha \text {-multiplication } x \dot{x} y=e^{\{\ln x \ln y\}}=x^{\ln y}=y^{\ln x} \\
\alpha \text {-division } x \dot{y}=e^{\{\ln x / \ln y\}}=x^{1 / \ln y} .
\end{gathered}
$$

Following Grosmann and Katz [8] we give the infinitely many $q_{r}$-arithmetic, of which the quadratic arithmetic and harmonic arithmetic are special cases for $r=2$ and $r=-1$, respectively. The function $q_{r}: \mathbb{R} \rightarrow \mathbb{R}_{q} \subseteq \mathbb{R}$ and its inverse $q_{r}^{-1}(x)$ are defined as follows:

$$
\begin{gathered}
q_{r}(x)= \begin{cases}x^{1 / r}, & x>0 \\
0, & x=0 \\
-(-x)^{1 / r}, & x<0,\end{cases} \\
q_{r}^{-1}(x)= \begin{cases}x^{r}, & x>0 \\
0, & x=0, \\
-(-x)^{r}, & x<0,\end{cases}
\end{gathered}
$$

$$
(r \in \mathbb{R} \backslash\{0\}) .
$$

If $r=1$ then the $q_{r}$-calculus is reduced to the classical calculus.
One can easily conclude that the $\alpha$-summation can be written as follows:

$$
\begin{aligned}
\sum_{k=1}^{n} x_{k} & =\alpha\left\{\sum_{k=1}^{n} \alpha^{-1}\left(x_{k}\right)\right\} \\
& =\alpha\left\{\alpha^{-1}\left(x_{1}\right)+\cdots+\alpha^{-1}\left(x_{n}\right)\right\} \quad \forall x_{k} \in \mathbb{R}_{\alpha} .
\end{aligned}
$$

Definition 1 (see [13]). Let $X=\left(X, d_{\alpha}\right)$ be an $\alpha$-metric space. Then the basic notions can be defined as follows:

(a) A sequence $x=\left(x_{k}\right)$ is a function from the set $\mathbb{N}$ into the set $\mathbb{R}_{\alpha}$. The $\alpha$-real number $x_{k}$ denotes the value of the function at $k \in \mathbb{N}$ and is called the $k$ th term of the sequence.

(b) A sequence $\left(x_{n}\right)$ in $X=\left(X, d_{\alpha}\right)$ is said to be $\alpha$ convergent if, for every given $\varepsilon>\dot{0}\left(\varepsilon \in \mathbb{R}_{\alpha}\right)$, there exist $n_{0}=n_{0}(\varepsilon) \in \mathbb{N}$ and $x \in X$ such that $d_{\alpha}\left(x_{n}, x\right)=\left|x_{n}-x\right|_{\alpha}<\varepsilon$ for all $n>n_{0}$ and is denoted by ${ }^{\alpha} \lim _{n \rightarrow \infty} x_{n}=x$ or $x_{n} \stackrel{\alpha}{\rightarrow} x$, as $n \rightarrow \infty$.

(c) A sequence $\left(x_{n}\right)$ in $X=\left(X, d_{\alpha}\right)$ is said to be $\alpha$-Cauchy if for every $\varepsilon>\dot{0}$ there is $n_{0}=n_{0}(\varepsilon) \in \mathbb{N}$ such that $d_{\alpha}\left(x_{n}, x_{m}\right) \dot{<} \varepsilon$ for all $m, n>n_{0}$.

Throughout this paper, we define the $p$ th $\alpha$-exponent $x^{p_{\alpha}}$ and $q$ th $\alpha$-root $x^{(1 / q)_{\alpha}}$ of $x \in \mathbb{R}_{\alpha}$ by

$$
\begin{gathered}
x^{2_{\alpha}}=x \dot{\times} x=\alpha\left\{\alpha^{-1}(x) \times \alpha^{-1}(x)\right\}=\alpha\left\{\left[\alpha^{-1}(x)\right]^{2}\right\} \\
x^{3_{\alpha}}=x^{2_{\alpha}} \dot{\times} x=\alpha\left\{\alpha^{-1}\left\{\alpha\left[\alpha^{-1}(x) \times \alpha^{-1}(x)\right]\right\} \times \alpha^{-1}(x)\right\} \\
=\alpha\left\{\left[\alpha^{-1}(x)\right]^{3}\right\} \\
\vdots \\
x^{p_{\alpha}}=x^{(p-1)_{\alpha}} \dot{\times} x=\alpha\left\{\left[\alpha^{-1}(x)\right]^{p}\right\},
\end{gathered}
$$

and $\sqrt[\alpha]{x}=x^{(1 / 2)_{\alpha}}=y$ provided there exists $y \in \mathbb{R}_{\alpha}$ such that $y^{2 \alpha}=x$.

2.1. *-Arithmetic. Suppose that $\alpha$ and $\beta$ be two arbitrarily selected generators and "star-" also be the ordered pair of types of arithmetic ( $\beta$-arithmetic, $\alpha$-arithmetic). The sets $\left(\mathbb{R}_{\beta}, \ddot{+}, \ddot{-}, \ddot{x}, \ddot{j}\right)$ and $\left(\mathbb{R}_{\alpha}, \dot{+}, \dot{-}, \dot{x}, \dot{j}\right)$ are complete ordered fields and beta(alpha)-generator generates beta(alpha)-arithmetic, respectively. Definitions given for $\beta$ arithmetic are also valid for $\alpha$-arithmetic. Also $\alpha$-arithmetic is used for arguments and $\beta$-arithmetic is used for values; in particular, changes in arguments and values are measured by $\alpha$-differences and $\beta$-differences, respectively.

Definition 2 (see [15]). (a) The *-limit of a function $f$, denoted by ${ }^{*} \lim _{x \rightarrow a} f(x)=b$, at an element $a$ in $\mathbb{R}_{\alpha}$ is, if it exists, the unique number $b$ in $\mathbb{R}_{\beta}$ such that 


$$
{ }^{*} \lim _{x \rightarrow a} f(x)=b \Longleftrightarrow \forall \varepsilon \ddot{>} \ddot{0}, \exists \delta \dot{>} \dot{0} \ni|f(x) \ddot{-} b|_{\beta} \ddot{<} \varepsilon \quad \forall \varepsilon \in \mathbb{R}_{\beta},|x \dot{-} a|_{\alpha} \dot{<} \text { for } x, \delta \in \mathbb{R}_{\alpha} \text {. }
$$

A function $f$ is $*$-continuous at a point $a$ in $\mathbb{R}_{\alpha}$ if and only if $a$ is an argument of $f$ and ${ }^{*} \lim _{x \rightarrow a} f(x)=f(a)$. When $\alpha$ and $\beta$ are the identity function $I$, the concepts of $*$-limit and $*$-continuity are identical with those of classical limit and classical continuity.

(b) The isomorphism from $\alpha$-arithmetic to $\beta$-arithmetic is the unique function $\iota$ (iota) which has the following three properties:

(i) $\iota$ is one to one.

(ii) $\iota$ is from $\mathbb{R}_{\alpha}$ onto $\mathbb{R}_{\beta}$.

(iii) For any numbers $u, v \in \mathbb{R}_{\alpha}$,

$$
\begin{aligned}
\iota(u+v) & =\iota(u) \ddot{+} \iota(v) ; \\
\iota(u \dot{-} v) & =\iota(u) \ddot{-} \iota(v) ; \\
\iota(u \dot{\times} v) & =\iota(u) \ddot{\times} \iota(v) ; \\
\iota(u \dot{\gamma} v) & =\iota(u) \ddot{l} \iota(v) ; \\
v & \neq \dot{0} ; u \leq v \Longleftrightarrow \iota(u) \ddot{\leq} \iota(v) .
\end{aligned}
$$

It turns out that $\iota(x)=\beta\left\{\alpha^{-1}(x)\right\}$ for every $x$ in $\mathbb{R}_{\alpha}$ and that $\iota(\dot{n})=\ddot{n}$ for every $\alpha$-integer $\dot{n}$. Since, for example, $u \dot{+} v=\iota^{-1}\{\iota(u) \ddot{+} \iota(v)\}$, it should be clear that any statement in $\alpha$-arithmetic can readily be transformed into a statement in $\beta$-arithmetic.

2.2. Non-Newtonian Complex Field and Some Inequalities. Let $a \in\left(\mathbb{R}_{\alpha}, \dot{+}, \dot{-}, \dot{x}, \dot{j}\right)$ and $b \in\left(\mathbb{R}_{\beta}, \ddot{+}, \ddot{-}, \ddot{x}, \ddot{j}\right)$ be arbitrarily chosen elements from corresponding arithmetic. Then the ordered pair $(a, b) \in \mathbb{R}_{\alpha} \times \mathbb{R}_{\beta} \subseteq \mathbb{R}^{2}$ is called a $*$ point. The set of all $*$-points is called the set of $*$-complex numbers and is denoted by $\mathbb{C}^{*}$; that is,

$$
\mathbb{C}^{*}:=\left\{z^{*}=(a, b) \mid a \in \mathbb{R}_{\alpha}, b \in \mathbb{R}_{\beta}\right\} .
$$

Define the binary operations addition $(\oplus)$ and multiplication $(\odot)$ of $*$-complex numbers $z_{1}^{*}=\left(a_{1}, b_{1}\right)$ and $z_{2}^{*}=\left(a_{2}, b_{2}\right)$ :

$$
\begin{aligned}
& \oplus: \mathbb{C}^{*} \times \mathbb{C}^{*} \longrightarrow \mathbb{C}^{*} \\
& \left(z_{1}^{*}, z_{2}^{*}\right) \longmapsto \\
& z_{1}^{*} \oplus z_{2}^{*}=\left(a_{1} \dot{+} a_{2}, b_{1} \ddot{+} b_{2}\right) \\
& \quad=\left(\alpha\left\{\alpha^{-1}\left(a_{1}\right)+\alpha^{-1}\left(a_{2}\right)\right\}, \beta\left\{\beta^{-1}\left(b_{1}\right)+\beta^{-1} b_{2}\right\}\right) \\
& \odot: \mathbb{C}^{*} \times \mathbb{C}^{*} \longrightarrow \mathbb{C}^{*}
\end{aligned}
$$

$$
\begin{aligned}
& \left(z_{1}^{*}, z_{2}^{*}\right) \longmapsto \\
& z_{1}^{*} \odot z_{2}^{*}=\left(\alpha\left\{\alpha^{-1}\left(a_{1}\right) \alpha^{-1}\left(a_{2}\right)-\beta^{-1}\left(b_{1}\right) \beta^{-1}\left(b_{2}\right)\right\},\right. \\
& \left.\quad \beta\left\{\alpha^{-1}\left(a_{1}\right) \beta^{-1}\left(b_{2}\right)+\beta^{-1}\left(b_{1}\right) \alpha^{-1}\left(a_{2}\right)\right\}\right)
\end{aligned}
$$

for all $a_{1}, a_{2} \in \mathbb{R}_{\alpha}$ and $b_{1}, b_{2} \in \mathbb{R}_{\beta}$.

Theorem 3 (see [15]). $\left(\mathbb{C}^{*}, \oplus, \odot\right)$ is a field.

Following Grossman and Katz [8] we can give the definition of $*$-distance and some applications with respect to the $*$-calculus.

Definition 4. Let $X$ be a nonempty set and let $d^{*}: X \times X \rightarrow$ $\mathbb{R}_{\beta}$ be a function such that, for all $x, y, z \in X$, the following axioms hold:

(NM1) $d^{*}(x, y)=\ddot{0}$ if and only if $x=y$,

(NM2) $d^{*}(x, y)=d^{*}(y, x)$,

(NM3) $d^{*}(x, y) \ddot{\leq} d^{*}(x, z) \ddot{+} d^{*}(z, y)$.

Then the pair $\left(X, d^{*}\right)$ and $d^{*}$ are called a non-Newtonian metric (*-metric) space and a $*$-metric on $X$, respectively.

The $*$-distance $d^{*}$ between two arbitrarily elements $z_{1}^{*}=$ $\left(a_{1}, b_{1}\right)$ and $z_{2}^{*}=\left(a_{2}, b_{2}\right)$ of the set $\mathbb{C}^{*}$ is defined by

$$
\begin{aligned}
& d^{*}\left(z_{1}^{*}, z_{2}^{*}\right) \\
& =\sqrt[\beta]{\left[\iota\left(a_{1}-a_{2}\right)\right]^{2 \beta} \ddot{+}\left(b_{1}-b_{2}\right)^{2 \beta}} \\
& =\beta\left\{\sqrt{\left[\alpha^{-1}\left(a_{1}\right)-\alpha^{-1}\left(a_{2}\right)\right]^{2}+\left[\beta^{-1}\left(b_{1}\right)-\beta^{-1}\left(b_{2}\right)\right]^{2}}\right\} .
\end{aligned}
$$

Up to now, we know that $\mathbb{C}^{*}$ is a field and the distance between two points in $\mathbb{C}^{*}$ is computed by the function $d^{*}$. Let $z^{*}=(a, b) \in \mathbb{C}^{*}$ be an arbitrary element. The distance function $d^{*}\left(z^{*}, 0^{*}\right)$ is called $*$-norm of $z^{*}$ and is denoted by $\ddot{\|} \cdot \ddot{\|}$. In other words, let $0^{*}=(\dot{0}, \ddot{0}) \in \mathbb{C}^{*}$; then

$$
\ddot{\|} z^{*} \ddot{\|}=d^{*}\left(z^{*}, 0^{*}\right)=\beta\left\{\sqrt{\left(\alpha^{-1}\{a\}\right)^{2}+\left(\beta^{-1}\{b\}\right)^{2}}\right\} .
$$

Moreover, for all $z_{1}^{*}, z_{2}^{*} \in \mathbb{C}^{*}$ we have $d^{*}\left(z_{1}^{*}, z_{2}^{*}\right)=\ddot{\|} z_{1}^{*} \ominus z_{2}^{*} \ddot{\|}$ where $d^{*}$ is the induced metric from $\|\cdot\|$ norm.

Theorem 5 (see [15]). $\left(\mathbb{C}^{*}, d^{*}\right)$ is a complete metric space, where $d^{*}$ is defined by (11). 
Corollary 6 (see [15]). $\mathbb{C}^{*}$ is a Banach space with the *-norm $\ddot{\|} \cdot \ddot{\|}$ which is defined by (12).

Definition 7. (a) Given a sequence $z_{k}^{*}=\left(a_{k}, b_{k}\right)$ of $*$-complex numbers, the formal notation

$$
\begin{aligned}
\sum_{k=0}^{\infty} z_{k}^{*} & =z_{0}^{*} \oplus z_{1}^{*} \oplus z_{2}^{*} \oplus \cdots \oplus z_{k}^{*} \oplus \cdots \\
& =\left(\sum_{\alpha=0}^{\infty} a_{k}, \sum_{k=0}^{\infty} b_{k}\right)
\end{aligned}
$$

is called an infinite series with $*$-complex terms, or simply complex $*$-series for all $k \in \mathbb{N}$. Also, for integers $n \in \mathbb{N}$, the finite $*$-sums $s_{n}^{*}={ }_{*} \sum_{k=0}^{n} z_{k}^{*}$ are called the partial sums of complex $*$-series. If the sequence $*$-converges to a complex number $s^{*}$ then we say that the series $*$-converges and write $s^{*}={ }_{*} \sum_{k=0}^{\infty} z_{k}^{*}$. The number $s^{*}$ is then called the $*$-sum of this series. If $\left(s_{n}\right) *$-diverges, we say that the series $*$-diverges or that it is $*$-divergent.

(b) A *-series $\sum_{k=0}^{\infty} z_{k}^{*}$ is said to $*$-converge absolutely if ${ }_{*} \sum_{k=0}^{\infty} \ddot{\|} z_{k}^{*} \ddot{\|}=\ell$ for some number $\ell \in \mathbb{R}_{\beta}$.

(c) Let $\left\{f_{n}(x)\right\}$ be a sequence of functions from $A \subseteq \mathbb{R}$ to $\mathbb{C}^{*}$ for each $n$. We say that $\left\{f_{n}(x)\right\}$ is uniformly $*$-convergent to $f$ on $A$ if and only if, for each $x \in A$ and for an arbitrary $\epsilon \ddot{>} \ddot{0}\left(\epsilon \in \mathbb{R}_{\beta}\right)$, there exists an integer $N=N(\epsilon, x)$ such that $d^{*}\left(f_{n}(x), f(x)\right) \ddot{<} \epsilon$ whenever $n>N$.

(d) The series $\sum_{*}^{\infty} f_{k}(x)$ is said to be uniformly *convergent to $f(x)$ on $A$ if, given any $\varepsilon \ddot{>} \ddot{0}$, there exists an integer $n_{0}(\varepsilon)$ such that

$$
d^{*}\left(* \sum_{k=0}^{n} f_{k}(x), f(x)\right) \ddot{<} \varepsilon \quad \text { whenever } n \geq n_{0}(\varepsilon) \text {. }
$$

Proposition 8 (see [15]). Let $p \geq 1$ and $z_{k}^{*}, t_{k}^{*} \in \mathbb{C}^{*}$ for $k \in$ $\{0,1,2,3, \ldots, n\}$. Then

$$
\begin{aligned}
& \left(\sum_{\beta=0}^{n} \ddot{\|} z_{k}^{*} \oplus t_{k}^{*} \ddot{\|}^{p_{\beta}}\right)^{(1 / p)_{\beta}} \\
& \ddot{\leq}\left(\sum_{\beta} \sum_{k=0}^{n} \ddot{\|} z_{k}^{*} \ddot{\|}^{p_{\beta}}\right)^{(1 / p)_{\beta}} \ddot{+}\left(\sum_{\beta=0}^{n} \ddot{\|} t_{k}^{*} \ddot{\|}^{p_{\beta}}\right)^{(1 / p)_{\beta}} .
\end{aligned}
$$

Remark 9. Let $z_{1}^{*}=\left(a_{1}, b_{1}\right), z_{2}^{*}=\left(a_{2}, b_{2}\right) \in \mathbb{C}^{*}$. Then the following statements hold:

(i) One has

$$
\begin{aligned}
z_{1}^{*} \oslash z_{2}^{*}=\frac{\left(a_{1}, b_{1}\right)}{\left(a_{2}, b_{2}\right)} \oslash \\
=\left(\alpha\left\{\frac{\alpha^{-1}\left(a_{1}\right) \alpha^{-1}\left(a_{2}\right)+\beta^{-1}\left(b_{1}\right) \beta^{-1}\left(b_{2}\right)}{\left(\alpha^{-1}\left(a_{2}\right)\right)^{2}+\left(\beta^{-1}\left(b_{2}\right)\right)^{2}}\right\},\right. \\
\left.\quad \beta\left\{\frac{\beta^{-1}\left(b_{1}\right) \alpha^{-1}\left(a_{2}\right)-\alpha^{-1}\left(a_{1}\right) \beta^{-1}\left(b_{2}\right)}{\left(\alpha^{-1}\left(a_{2}\right)\right)^{2}+\left(\beta^{-1}\left(b_{2}\right)\right)^{2}}\right\}\right) .
\end{aligned}
$$

(ii) Let $\alpha$ and $\beta$ be the same generators. Then

$$
\begin{aligned}
z_{1}^{*} \odot \overline{z^{*}}=\left(a_{1}, b_{1}\right) \odot\left(a_{1}, \ddot{-} b_{1}\right) \\
\quad=\left(\alpha\left\{\left(\alpha^{-1}\left(a_{1}\right)\right)^{2}+\left(\beta^{-1}\left(b_{1}\right)\right)^{2}\right\}, \ddot{0}\right)=\ddot{\|} z^{*} \ddot{\|}^{2_{\beta}} .
\end{aligned}
$$

Definition 10. Given a point $x_{0} \in X$. Then, for a positive $\beta$ real number $r$,

$$
\begin{aligned}
& B^{*}\left(x_{0} ; r\right)=\left\{x \in X \mid d^{*}\left(x, x_{0}\right) \ddot{<} r\right\}, \\
& B^{*}\left[x_{0} ; r\right]=\left\{x \in X \mid d^{*}\left(x, x_{0}\right) \ddot{\leq} r\right\}
\end{aligned}
$$

are $*$-neighborhood (or $*$-open (closed) ball) of centre $x_{0}$ and radius $r$, respectively.

We see that an $*$-open ball of radius $r$ is the set of all points in $X$ whose beta-distance from the center of the ball is less than $r$ and we say directly from the definition that every *neighborhood of $x_{0}$ contains $x_{0}$; in other words, $x_{0}$ is a point of each of its $*$-neighborhoods.

Definition 11. Let $\left(X, d^{*}\right)$ be a $*$-metric space. Then the followings are valid:

(i) $G \subset X$ is called *-open set if and only if every point of $G$ has a *-neighborhood contained in $G$. Also $G \subset X$ is called $*$-closed set if and only if its complement is *-open.

(ii) The $*$-interior $G^{o}$ is the largest $*$-open set contained in $G$ and the $*$-closure $\bar{G}$ is the smallest $*$-closed set contained in $G$.

Definition 12 (usual *-topology). Consider the set of *complex numbers with

$$
\tau=\left\{S \subseteq \mathbb{C}^{*} \mid \forall x \in S, \exists r \ddot{>} 0 \ni B^{*}\left(x_{0} ; r\right) \subseteq S\right\}
$$

for all $x_{0} \in \mathbb{C}^{*}$ and $r \in \mathbb{R}_{\beta}$. Then $\left(\mathbb{C}^{*}, \tau\right)$ is a topological space and is called $*$-usual topology on $\mathbb{C}^{*}$.

Definition 13. (i) A topological $*$-vector (linear) space $X$ is a *-vector space (see [17]) over the topological field that endowed with a topology such that $*$-vector addition and scalar multiplication are $*$-continuous functions.

(ii) A topological $*$-vector space is called $*$-normable if the topology of the space can be induced by a $*$-norm.

Definition 14. A sequence space $\lambda$ with a $*$-linear topology is called a $* \mathrm{~K}$-space provided each of the maps $p_{i}: \lambda \rightarrow \mathbb{C}^{*}$ defined by $p_{i}(x)=x_{i}$ is $*$-continuous for all $i \in \mathbb{N}$. A $* \mathrm{~K}$ space is called a $* \mathrm{FK}$-space provided $\lambda$ is a complete linear $*$-metric space (see [15]). An $* \mathrm{FK}$-space whose topology is $*$-normable is called a $* \mathrm{BK}$-space.

Definition 15 ( $p$-*normed space). Let $X$ be a real or complex *-linear space and let $\ddot{\|} \cdot \ddot{\|}_{p}$ be a function from $X$ to the set $\mathbb{R}_{\beta}^{+}$and $p>0$. Then the pair $\left(X, \ddot{\|} \cdot \ddot{\|}_{p}\right)$ is called a $p$-*normed space and $\ddot{\|} \cdot \ddot{\|}_{p}$ is a $p$-*norm for $X$, if the following axioms are satisfied for all elements $x, y \in X$ and for all scalars $\lambda$ : 
(N1) $\ddot{\|} x \ddot{\|}_{p}=\ddot{0} \Leftrightarrow x=\theta^{*}\left(\theta^{*}=(\dot{0}, \ddot{0})\right)$,

(N2) $\ddot{\|} \lambda \odot x \ddot{\|}_{p}=\ddot{\|} \lambda \ddot{\|}{ }^{p_{\beta}} \ddot{x} \ddot{\|} x \ddot{\|}_{p}(\ddot{\|} \lambda \ddot{\|}$ is complex modulus),

(N3) $\ddot{\|} x \oplus y \ddot{\|}_{p} \ddot{\leq} \ddot{\|} x \ddot{\|}_{p} \ddot{+} \ddot{\|} y \ddot{\|}_{p}$.

Definition 16. (i) A *-linear map or *-linear operator $T$ between real (or complex) *-linear spaces $X, Y$ is a function $T: X \rightarrow Y$ such that $T(\lambda \dot{\times} x \dot{+} \mu \dot{\times} y)=\lambda \dot{\times} T x+\mu \dot{\times} T y$ for all $\lambda, \mu \in \mathbb{R}_{\alpha}$ and similarly $T\left(\lambda^{*} \odot x \oplus \mu^{*} \odot y\right)=\lambda^{*} \odot T x \oplus \mu^{*} \odot T y$ for all $\lambda^{*}, \mu^{*} \in \mathbb{C}^{*}$ and $x, y \in X$.

(ii) Let $X$ and $Y$ be two $*$-normed linear spaces. A *linear map $T: X \rightarrow Y$ is $*$-bounded if there is a constant $M \ddot{\geq} 0$ such that $\ddot{\|} T x \ddot{\|}_{Y} \ddot{\leq} M \ddot{\times} \| \ddot{\|}_{X}$ for all $x \in X$. We denote the set of all $*$-linear maps $T: X \rightarrow Y$ by $\mathscr{L}(X, Y)$ and the set of all $*$-bounded linear maps by $\mathscr{B}(X, Y)$.

\section{Non-Newtonian Infinite Matrices}

Let $w^{*}$ denote the set of all $*$-complex sequences $x=\left(x_{k}\right)_{k=0}^{\infty}$. As usual, we write $\ell_{\infty}^{*}, c^{*}, c_{0}^{*}$ for the sets of all *-bounded, *convergent, $*$-null sequences and

$$
\ell_{p}^{*}=\left\{z^{*}=\left(z_{k}^{*}\right) \in \omega^{*}: \sum_{k=1}^{\infty} \ddot{\|} z_{k}^{*} \ddot{\|}^{\ddot{p}}<\infty\right\}
$$

for $1 \leq p<\infty$.

A non-Newtonian infinite matrix $A=\left(a_{i j}^{*}\right)$ of $*$-complex numbers is defined by a function $A$ from the set $\mathbb{N} \times \mathbb{N}$ into $\mathbb{C}^{*}$. The addition $(\oplus)$ and scalar multiplication $(\odot)$ of the infinite matrices $A=\left(a_{i j}^{*}\right)=\left(\epsilon_{i j}, \delta_{i j}\right)$ and $B=\left(b_{i j}^{*}\right)=\left(\mu_{i j}, \eta_{i j}\right)$ are defined by

$$
\begin{aligned}
A & \oplus B=\left(a_{i j}^{*} \oplus b_{i j}^{*}\right) \\
& =\left(\epsilon_{i j}, \delta_{i j}\right) \oplus\left(\mu_{i j}, \eta_{i j}\right) \\
& =\left(\epsilon_{i j}+\mu_{i j}, \delta_{i j} \ddot{+} \eta_{i j}\right) \\
& =\left(\alpha\left\{\alpha^{-1}\left(\epsilon_{i j}\right)+\alpha^{-1}\left(\mu_{i j}\right)\right\}, \beta\left\{\beta^{-1}\left(\delta_{i j}\right)+\beta^{-1}\left(\eta_{i j}\right)\right\}\right), \\
\lambda^{*} \odot A=\left(\lambda^{*} \odot a_{i j}^{*}\right) & \\
& =(\dot{\lambda}, \ddot{\lambda}) \odot\left(\epsilon_{i j}, \delta_{i j}\right) \\
& =\left(\alpha\left\{\lambda \epsilon_{i j}-\lambda \delta_{i j}\right\}, \beta\left\{\lambda \epsilon_{i j}+\lambda \delta_{i j}\right\}\right), \quad\left(\lambda^{*}=(\dot{\lambda}, \ddot{\lambda}) \in \mathbb{C}^{*}\right),
\end{aligned}
$$

where $\varepsilon_{i j}, \mu_{i j} \in \mathbb{R}_{\alpha}$ and $\delta_{i j}, \eta_{i j} \in \mathbb{R}_{\beta}$ for all $i, j \in \mathbb{N}$. Also, the product $(A \odot B)_{i j}$ of $A=\left(a_{i j}^{*}\right)$ and $B=\left(b_{i j}^{*}\right)$ can be interpreted as

$$
\begin{aligned}
= & \sum_{k=0}^{\infty} a_{i k}^{*} \odot b_{k j}^{*} \\
= & \left(\alpha\left\{\sum_{k=0}^{\infty}\left(\alpha^{-1}\left(\varepsilon_{i k}\right) \alpha^{-1}\left(\mu_{k j}\right)-\beta^{-1}\left(\delta_{i k}\right) \beta^{-1}\left(\eta_{k j}\right)\right)\right\},\right. \\
& \left.\beta\left\{\sum_{k=0}^{\infty}\left(\alpha^{-1}\left(\varepsilon_{i k}\right) \beta^{-1}\left(\eta_{k j}\right)+\beta^{-1}\left(\delta_{i k}\right) \alpha^{-1}\left(\mu_{k j}\right)\right)\right\}\right)
\end{aligned}
$$

provided the series on the right hand side converge. On the other hand the series on the right hand side of (22) converges if and only if

$$
\begin{aligned}
& \sum_{k=0}^{\infty}\left(\alpha^{-1}\left(\varepsilon_{i k}\right) \alpha^{-1}\left(\mu_{k j}\right)-\beta^{-1}\left(\delta_{i k}\right) \beta^{-1}\left(\eta_{k j}\right)\right), \\
& \sum_{k=0}^{\infty}\left(\alpha^{-1}\left(\varepsilon_{i k}\right) \beta^{-1}\left(\eta_{k j}\right)+\beta^{-1}\left(\delta_{i k}\right) \alpha^{-1}\left(\mu_{k j}\right)\right)
\end{aligned}
$$

are convergent classically. However the series (22) may *diverge for some, or all, values of $i$, $j$; the product $A \odot B$ may not exist.

Let $\mu_{1}^{*}, \mu_{2}^{*} \subset w^{*}$, and $A=\left(a_{n k}^{*}\right)=\left(\varepsilon_{n k}, \delta_{n k}\right)$ be an infinite matrix of $*$-complex numbers. Then we say that $A$ defines a matrix mapping from $\mu_{1}^{*}$ into $\mu_{2}^{*}$ and denote it by writing $A$ : $\mu_{1}^{*} \rightarrow \mu_{2}^{*}$, if for every sequence $z=\left(z_{k}^{*}\right) \in \mu_{1}^{*}$ the sequence $A \odot z=\left\{(A z)_{n}\right\}$, the $A$-transform of $z$, exists and is in $\mu_{2}^{*}$. In this way, we transform the sequence $z=\left(z_{k}^{*}\right)=\left(\mu_{k}, \eta_{k}\right)$ with $\mu_{k} \in \mathbb{R}_{\alpha}$ and $\eta_{k} \in \mathbb{R}_{\beta}$, into the sequence $\left\{(A z)_{n}\right\}$ by

$$
\begin{aligned}
& \left\{(A z)_{n}\right\}=\sum_{*} a_{n k}^{*} \odot z_{k}^{*} \\
& =\left(\alpha\left\{\sum_{k=0}^{\infty}\left(\alpha^{-1}\left(\varepsilon_{n k}\right) \alpha^{-1}\left(\mu_{k}\right)-\beta^{-1}\left(\delta_{n k}\right) \beta^{-1}\left(\eta_{k}\right)\right)\right\},\right. \\
& \left.\quad \beta\left\{\sum_{k=0}^{\infty}\left(\alpha^{-1}\left(\varepsilon_{n k}\right) \beta^{-1}\left(\eta_{k}\right)+\beta^{-1}\left(\delta_{n k}\right) \alpha^{-1}\left(\mu_{k}\right)\right)\right\}\right)
\end{aligned}
$$

for all $k, n \in \mathbb{N}$. Thus, $A \in\left(\mu_{1}^{*}: \mu_{2}^{*}\right)$ if and only if the series on the right side of $(24) *$-converges for each $n \in \mathbb{N}$ and every $z \in \mu_{1}^{*}$, and we have $A \odot z=\left\{(A z)_{n}\right\}_{n \in \mathbb{N}} \in \mu_{2}^{*}$ for all $z \in \mu_{1}^{*}$. A sequence $z$ is said to be $A$-summable to $\gamma$ if $A \odot z *$-converges to $\gamma \in \mathbb{C}^{*}$ which is called $A-{ }^{*} \lim$ of $z$.

The Cesàro transform of a sequence $z=\left(z_{k}^{*}\right) \in w^{*}$ is given by $C_{1}^{*} \odot z=\left\{\left(C_{1}^{*} z\right)_{n}\right\}_{n \in \mathbb{N}}$. Now, following Example 17 we may state the Cesàro summability with respect to the nonNewtonian calculus which is analogous to the classical Cesàro summable.

Example 17. Define the matrix $C_{1}^{*}=\left(c_{n k}^{*}\right)$ by

$$
\begin{aligned}
& c_{n k}^{*}(\alpha, \beta) \\
& = \begin{cases}\left(\frac{1}{n+1}\right)^{*}=\left(\alpha\left(\frac{1}{n+1}\right), \beta\left(\frac{1}{n+1}\right)\right), & 0 \leq k \leq n, \\
0^{*}=(\alpha(0), \beta(0)), & k>n .\end{cases}
\end{aligned}
$$

If we choose the generator functions as $\alpha=\exp$ and $\beta=\exp$, then the infinite matrix can be written as follows: 


$$
c_{n k}^{*}(\exp , \exp )=\left(\begin{array}{cccccc}
(e, e) & (1,1) & \ldots & \cdots & \ldots & \ldots \\
(\sqrt{e}, \sqrt{e}) & (\sqrt{e}, \sqrt{e}) & (1,1) & \ldots & \ldots & \ldots \\
(\sqrt[3]{e}, \sqrt[3]{e}) & (\sqrt[3]{e}, \sqrt[3]{e}) & (\sqrt[3]{e}, \sqrt[3]{e}) & (1,1) & \ldots & \ldots \\
\vdots & \vdots & \vdots & \ddots & \vdots & \vdots \\
(\sqrt[n+1]{e}, \sqrt[n+1]{e}) & (\sqrt[n+1]{e}, \sqrt[n+1]{e})(\sqrt[n+1]{e}, \sqrt[n+1]{e}) & \cdots & (\sqrt[n+1]{e}, \sqrt[n+1]{e}) & \cdots \\
\vdots & \vdots & \vdots & \ldots & \vdots & \ddots
\end{array}\right) .
$$

The $C_{1}^{*}$-transform of a sequence $x=\left(x_{k}^{*}\right)=\left(a_{k}, b_{k}\right)$ is the sequence $y=\left(y_{n}^{*}\right)$ defined by

$$
\begin{aligned}
y_{n}^{*} & =\left(C_{1}^{*} \odot x\right)_{n}=\sum_{*=1}^{n} \frac{x_{k}^{*}}{\dot{n}} \oslash \\
& =\left(\alpha \sum_{k=1}^{n} \frac{a_{k}}{\dot{n}} \cdot, \quad \sum_{k=1}^{n} \frac{b_{k}}{\ddot{n}}:\right) \\
& =\left(\alpha\left\{\frac{1}{n} \sum_{k=1}^{n} \alpha^{-1}\left(a_{k}\right)\right\}, \beta\left\{\frac{1}{n} \sum_{k=1}^{n} \beta^{-1}\left(b_{k}\right)\right\}\right),
\end{aligned}
$$

where ( $\oslash$ is $*$-complex division), $a_{k} \in \mathbb{R}_{\alpha}$, and $b_{k} \in \mathbb{R}_{\beta}$ for all $k \in \mathbb{N}$. Taking $\alpha=\exp$ and $\beta=\exp$, we obtain $C_{1}^{*}$-transform of $x=\left(x_{k}^{*}\right)=\left(a_{k}, b_{k}\right)$ as follows:

$$
\begin{aligned}
y_{n}^{*} & =\left(C_{1}^{*} \odot x\right)_{n} \\
& =\left(\exp \left\{\frac{1}{n} \sum_{k=1}^{n} \ln \left(a_{k}\right)\right\}, \exp \left\{\frac{1}{n} \sum_{k=1}^{n} \ln \left(b_{k}\right)\right\}\right) \\
& =\left(\left(a_{1} \cdots a_{n}\right)^{1 / n},\left(b_{1} \cdots b_{n}\right)^{1 / n}\right), \\
& \left(a_{n}, b_{n} \in \mathbb{R}_{\exp }\right) .
\end{aligned}
$$

\section{Matrix Transformations Related to the Strong Cesàro *-Summability}

Let $e$ and $e^{(n)}(n=0,1, \ldots)$ be the sequences with $e_{k}=1^{*}$ for all $k \in \mathbb{N}$ and $e_{n}^{(n)}=1^{*}$ and $e_{k}^{(n)}=0^{*}$ for $k \neq n$. Also, if $X$ is $\mathrm{a} * \mathrm{BK}$-space and $a \in \omega^{*}$ then we write $\|a\|_{\ddagger}=\sup \left\{\ddot{\|}{ }_{*} \sum a_{k} \odot\right.$ $x_{k} \ddot{\|}: \ddot{\|} x \ddot{\|}=\ddot{1}$ \}. Moreover, $\mathrm{a} * \mathrm{BK}$-space $X \supset \emptyset$ is said to have $*$ AK if every sequence $x=\left(x_{k}\right)_{k=1}^{\infty} \in X$ has a unique representation $x={ }_{*} \sum_{k=1}^{\infty} x_{k} \odot e^{(k)}$. If $X$ is a subset of $w^{*}$ then $X^{\beta}=\left\{a \in \omega^{*}: a \odot x \in c s^{*}\right.$ for all $\left.x \in X\right\}$ is called the $\beta$-dual of $X$.

Throughout the text we use the notation $\left[C_{1}^{*}\right]_{p}$ for strong Cesàro $*$-summability of order 1 and index $p$. Maddox [19] introduced and studied the sets of sequences that are strongly summable and bounded with index $p,(1 \leq p<\infty)$ by the Cesàro method of order one. By taking into account the sets $\omega_{0}^{p}(\alpha, \beta), \omega^{p}(\alpha, \beta)$, and $\omega_{\infty}^{p}(\alpha, \beta)$ of sequences that are strongly $*$-summable to zero, $*$-summable, and $*$-bounded of index $p \geq 1$ are defined by

$$
\begin{aligned}
\omega_{0}^{p}(\alpha, \beta) & =\left\{x_{k}=\left(a_{k}, b_{k}\right) \in \omega^{*}:{ }^{\beta} \lim _{n \rightarrow \infty} \sum_{k=1}^{n} \frac{\ddot{\|} x_{k} \ddot{\|}^{p_{\beta}}}{\ddot{n}}:=\ddot{0}\right\} \quad\left(a_{k} \in \mathbb{R}_{\alpha}, b_{k} \in \mathbb{R}_{\beta}\right) \\
& =\left\{\left(a_{k}, b_{k}\right) \in \omega^{*}: \lim _{n \rightarrow \infty} \beta\left\{\frac{1}{n} \sum_{k=1}^{n}\left[\left(\alpha^{-1}\left\{a_{k}\right\}\right)^{2}+\left(\beta^{-1}\left\{b_{k}\right\}\right)^{2}\right]^{p / 2}\right\}=\ddot{0}\right\}, \\
\omega^{p}(\alpha, \beta) & =\left\{x_{k}=\left(a_{k}, b_{k}\right) \in \omega^{*}:{ }^{\beta} \lim _{n \rightarrow \infty} \sum_{k=1}^{n} \frac{\ddot{\|} x_{k} \Theta \ell \ddot{\|} p^{p_{\beta}}}{\ddot{n}}:=\ddot{0} \text { for some } \ell \in \mathbb{C}^{*}\right\} \\
& =\left\{\left(a_{k}, b_{k}\right) \in \omega^{*}: \lim _{n \rightarrow \infty} \beta\left\{\frac{1}{n} \sum_{k=1}^{n}\left[\left(\alpha^{-1}\left\{a_{k}\right\}-\alpha^{-1}\left\{\ell_{1}\right\}\right)^{2}+\left(\beta^{-1}\left\{b_{k}\right\}-\beta^{-1}\left\{\ell_{2}\right\}\right)^{2}\right]^{p / 2}\right\}=\ddot{0}, \ell=\left(\ell_{1}, \ell_{2}\right) \in \mathbb{C}^{*}\right\}, \\
\omega_{\infty}^{p}(\alpha, \beta) & =\left\{x_{k}=\left(a_{k}, b_{k}\right) \in \omega^{*}: \sup _{n \in \mathbb{N}} \sum_{k=1}^{n} \frac{\ddot{\|} x_{k} \ddot{\|} p^{p_{\beta}}: \ddot{<} \infty}{\ddot{n}}\right\}=\left\{\left(a_{k}, b_{k}\right) \in \omega^{*}: \sup _{n \in \mathbb{N}} \beta\left\{\frac{1}{n} \sum_{k=0}^{n}\left[\left(\alpha^{-1}\left\{a_{k}\right\}\right)^{2}+\left(\beta^{-1}\left\{b_{k}\right\}\right)^{2}\right]^{p / 2}\right\} \ddot{<\infty}\right\} .
\end{aligned}
$$


For instance,

(i) $\omega_{0}^{p}\left(\exp , q_{r}\right)=\left\{\left(a_{k}, b_{k}\right) \in \omega^{*}: \lim _{n \rightarrow \infty}\{(1 /\right.$ n) $\left.\left.\sum_{k=1}^{n}\left(\ln ^{2}\left(a_{k}\right)+\left(b_{k}\right)^{2 r}\right)^{p / 2}\right\}^{1 / r}=0\right\},\left(a_{k} \in \mathbb{R}_{\exp }, b_{k} \in\right.$ $\left.\mathbb{R}_{q}, r \in \mathbb{R}^{+}, p \geq 1\right)$;

(ii) $\omega^{p}\left(q_{r}, \exp \right)=\left\{\left(a_{k}, b_{k}\right) \in \omega^{*}: \lim _{n \rightarrow \infty}\{(1 /\right.$ n) $\left.\left.\sum_{k=1}^{n}\left[\left(\left(a_{k}\right)^{r}-\left(\ell_{1}\right)^{r}\right)^{2}+\ln ^{2}\left(b_{k} / l_{2}\right)\right]^{p / 2}\right\}=0\right\},\left(a_{k}, \ell_{1} \in\right.$ $\left.\mathbb{R}_{q} ; b_{k}, \ell_{2} \in \mathbb{R}_{\exp }\right)$.

Theorem 18. The sets $\omega_{0}^{p}(\alpha, \beta), \omega^{p}(\alpha, \beta)$, and $\omega_{\infty}^{p}(\alpha, \beta)$ are complete $*$-metric spaces over the field $\mathbb{C}^{*}$ with the metric $d_{p}^{(\alpha, \beta)}$ defined by

$$
\begin{aligned}
& d_{p}^{(\alpha, \beta)}(x, y) \\
& := \begin{cases}\sup _{n \in \mathbb{N}} \beta \sum_{k=1}^{n} \frac{\ddot{\|} x_{k} \odot y_{k} \ddot{\|}^{p_{\beta}}}{\ddot{n}}:, & 0<p<1, \\
\sup _{n \in \mathbb{N}}\left\{\sum_{k=1}^{n} \frac{\ddot{\|} x_{k} \odot y_{k} \ddot{\|}^{p_{\beta}}}{\ddot{n}}:\right\}^{(1 / p)_{\beta}}, & 1 \leq p<\infty .\end{cases}
\end{aligned}
$$

Proof. The proof is a routine verification and hence omitted.

Corollary 19. The sets $\omega_{0}^{p}(\alpha, \beta), \omega^{p}(\alpha, \beta)$, and $\omega_{\infty}^{p}(\alpha, \beta)$ are Banach spaces with the induced metric $d_{p}^{(\alpha, \beta)}$ from the corresponding norm $\| x \ddot{\|}_{\dagger}$ defined by

$$
\|z\|_{\mathscr{M}_{p}^{(\alpha, \beta)}}= \begin{cases}\sum_{\gamma=0}^{\infty} \ddot{2}^{\nu_{\beta}} \ddot{\times} \max _{\nu} \ddot{\|} z_{k} \ddot{\|}, & p=1, \\ \sum_{\beta}^{\infty} \ddot{2}^{(v / p)_{\beta}} \ddot{\times}\left(\sum_{\gamma} \ddot{\|} z_{k} \ddot{\|}^{q_{\beta}}\right)^{(1 / q)_{\beta}}, \quad\left(1<p<\infty ; q=\frac{p}{(p-1)}\right), & \forall z \in \mathscr{M}_{p}^{(\alpha, \beta)} .\end{cases}
$$

where

$$
\begin{aligned}
& \|x\|_{\omega_{\infty}^{p}(\alpha, \beta)}=\sup _{\nu \in \mathbb{N}}\left(\sum_{\gamma} \frac{\ddot{\|} x_{k} \ddot{\|}^{p_{\beta}}}{2^{\nu_{\beta}}}:\right)^{(1 / p)_{\beta}}, \\
& \|x\|_{\omega_{\infty}^{p}(\alpha, \beta)}^{\dagger}=\sup _{n \in \mathbb{N}}\left(\sum_{\beta}^{n} \frac{\ddot{\|} x_{k} \ddot{\|}^{p_{\beta}}}{\ddot{n}}:\right)^{(1 / p)_{\beta}} .
\end{aligned}
$$

Proof. The proof is straightforward (see [20]).

First we give the $\beta$-duals of the spaces $\omega_{0}^{p}(\alpha, \beta), \omega^{p}(\alpha, \beta)$, and $\omega_{\infty}^{p}(\alpha, \beta)$. We prefer the notation $\sum_{v}$ and $\max _{v}$ for the sum and maximum taken over all indices $k$ with $2^{\nu} \leq k \leq$ $2^{\nu+1}-1$ and put

$$
\mathscr{M}_{p}^{(\alpha, \beta)}=\left\{z \in \omega^{*}:\|z\|_{\mathscr{M}_{p}} \ddot{<} \infty\right\}
$$

In particular, we have

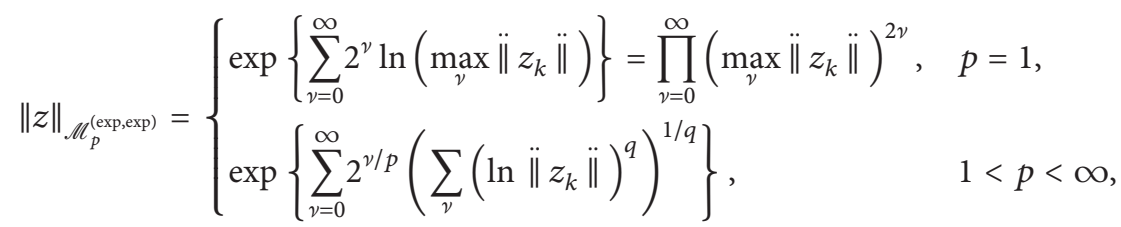

for all $z_{k}=\left(a_{k}, b_{k}\right) \in \omega^{*}$ where $a_{k}, b_{k} \in \mathbb{R}_{\exp }=(0, \infty)$.

Now, with regard to notation, for any matrix $A=\left(a_{n k}^{*}\right)$ we write, for $1 / p+1 / q=1, p \geq 1$,

$$
A_{\nu}^{p}(n)=\left({ }_{\beta} \sum_{\nu} \ddot{\|} a_{n k}^{*} \ddot{\|}^{q_{\beta}}\right)^{(1 / q)_{\beta}}
$$

and the case $p=1$ of (36) is interpreted as

$$
A_{v}^{1}(n)=\max _{v} \ddot{\|} a_{n k}^{*} \ddot{\|}
$$

Remark 21. Let $Y$ be a $*$-normed space and let $T$ be a $*$-linear operator on the $p$-*normed space $X$ into $Y$. Then it is easy to see that $T$ is $*$-continuous on $X$ if and only if there is a positive constant $M \in \mathbb{R}_{\beta}$ such that $\ddot{\|} T x \ddot{\|}_{Y} \ddot{\leq} M \ddot{\times} \ddot{\|} x \ddot{\|}_{X}^{(1 / p)_{\beta}}$ on $X$. The $*$-norm on the left is the norm in $Y$ and that on the right is $p-*$ norm in $X$. It is also seen that Banach-Steinhaus theorem in the non-Newtonian sense holds in a $p$-*normed space defined in Definition 15.

Also one can immediately conclude that $\left[C_{1}^{*}\right]_{p}$ is *complete in the metric generated by the $*$-norm. For simplicity we denote $\left[C_{1}^{*}\right]_{p}$ by $\Omega$, so that, in the case $p \geq 1, \Omega$ 
is a Banach space and, in the case $0<p<1, \Omega$ is a complete $p$-*normed space. The dual space of $\Omega$, that is, the space of continuous $*$-linear functionals on $\Omega$, will be denoted by $\Omega^{*}$.

Corollary 22 (cf. [19] (Banach-Steinhaus)). If $\left(T_{n}\right)$ is a sequence of continuous *-linear operators on a $p$ *normed space $X$ into a *-normed space $Y$ such that ${ }^{\beta} \lim \sup _{n} \ddot{\|} T_{n} x \ddot{\|}_{Y} \ddot{<} \infty$ on a second category set in $X$, then $\sup _{n}\left\|T_{n}\right\| \ddot{<} \infty$. It is important to remark that $\left\|T_{n}\right\|$ denotes the norm of $T_{n}$, that is, $\left\|T_{n}\right\|=\sup _{n} \ddot{\|} T_{n} x \ddot{\|}_{Y} \ddot{\times} \ddot{\|} x \ddot{\|}_{X}^{(-1 / p)_{\beta}}$, the supremum being taken over all non- $\beta$-zero elements of $X$.

The next theorem characterizes all infinite matrices $A$ which map the space $\left[C_{1}^{*}\right]_{p}$ into the space $c^{*}$ of convergent sequences of $*$-complex numbers.

Theorem 23. $(A z)_{n}$ is defined for each $n$ and the sequence $\left\{(A z)_{n}\right\}$ is *-convergent, whenever $z \in \Omega$, if and only if,

(a) for $0<p<1$

$$
\begin{aligned}
& \text { (i) } d^{*}\left(a_{n k}^{*}, \xi_{k}\right)=\ddot{0} \quad(n \rightarrow \infty, k \text { fixed }), \\
& \text { (ii) } M=\sup _{n}\left\{_{\beta} \sum_{\nu=0}^{\infty} \ddot{2}^{(v / p)_{\beta}} \ddot{\times} A_{\nu}^{1}(n)\right\} \ddot{<} \infty \text {; }
\end{aligned}
$$

(b) for $p \geq 1$,

$$
\begin{aligned}
& \text { (i) as in (a) above, } \\
& \text { (ii) } M={ }_{\beta} \sum_{\nu=0}^{\infty} \ddot{2}^{(v / p)_{\beta}} \ddot{\times} A_{\nu}^{p}(n) \ddot{<} \infty, \\
& \text { (iii) } d^{*}\left({ }_{*} \sum_{k=0}^{\infty} a_{n k}^{*}, \xi\right)=\ddot{0},(n \rightarrow \infty) .
\end{aligned}
$$

Proof. The proofs of necessity condition of (i) and (iii) and the sufficiency of the conditions are routine verification and hence omitted.

Firstly we prove the sufficiency when $0<p<1$, leaving the necessities (ii) and (ii) ${ }^{\prime}$ to the next. Now, consider that the condition (ii) holds. Then the series defining $(A z)_{n}$ is absolutely $*$-convergent (see Definition 7) for each $n \in \mathbb{N}$. By using the known inequality we have

$$
\begin{aligned}
& \sum_{k=1}^{\infty} \ddot{\|} a_{n k}^{*} \odot z_{k} \ddot{\|} \\
& =\sum_{\beta} \sum_{\gamma=0}^{\infty} \sum_{\nu} \ddot{\|} a_{n k}^{*} \odot z_{k} \ddot{\|} \\
& \ddot{\leq} \sum_{\beta=0}^{\infty}\left(\sum_{\beta} \ddot{\|} a_{n k}^{*} \odot z_{k} \ddot{\|}^{p_{\beta}}\right)^{(1 / p)_{\beta}} \\
& \ddot{\leq} \sum_{\beta=0}^{\infty} A_{\nu}^{1}(n) \ddot{x} \ddot{2}^{(\nu / p)_{\beta}} \ddot{x} \ddot{\|} z \ddot{\|}_{p}^{1 / p} \ddot{\leq} M \ddot{x} \ddot{\|} z \ddot{\|}_{p}^{1 / p} \ddot{<} \infty .
\end{aligned}
$$

Again for $0<p<1$ we will show that the conditions (i) and (ii) imply (iii). In fact we will prove that (ii) which implies $\sum_{k=0}^{\infty} \ddot{\|} a_{n k}^{*} \ddot{\|}$ is uniformly $*$-convergent (see Definition 7) and this together with (i) gives

$$
{ }^{*} \lim _{n} \sum a_{n k}^{*}=\sum \xi_{k} .
$$

To prove uniformly $*$-convergent, by using the conjugate numbers $p$ and $q$ for $0<p<1$, then, for any positive integer $n_{0}$ and for any $m \geq 2^{n_{0}}$, we obtain

$$
\begin{aligned}
\beta \sum_{k=m}^{\infty} \ddot{\|} a_{n k}^{*} \ddot{\|} & \ddot{\leq} \sum_{\gamma=n_{0}}^{\infty} \sum_{\nu} \ddot{\|} a_{n k}^{*} \ddot{\|} \\
& \ddot{\leq} \sum_{\beta=n_{0}}^{\infty} A_{\nu}^{1}(n) \ddot{\times} \ddot{2}^{v_{\beta}} \ddot{\leq} M \ddot{\times} \ddot{2}^{\left(n_{0} / q\right)_{\beta}} .
\end{aligned}
$$

Thus,

$$
\begin{aligned}
& { }_{*} \sum a_{n k}^{*} \odot z_{k} \\
& ={ }_{*} \sum \xi_{k} \odot z_{k} \oplus\left[\ell \odot_{*} \sum\left(a_{n k}^{*} \ominus \xi_{k}\right)\right] \oplus{ }_{*} \sum\left(a_{n k}^{*} \ominus \xi_{k}\right)\left(z_{k} \ominus \ell\right)
\end{aligned}
$$

holds where $z_{k} \quad \rightarrow \quad \ell$, and (i)-(ii) imply ${ }_{\beta} \sum_{\nu=0}^{\infty} \ddot{2}^{(\nu / p)_{\beta}} \ddot{x} \max _{v} \ddot{\|} \xi_{k} \ddot{\|} \ddot{\leq} M$, and we deduce that $\sum_{*} \sum \xi_{k} \odot z_{k}$ is absolutely $*$-convergent and the last sum in (41) goes to $\ddot{0}$ as $n \rightarrow \infty$. From (39),

$$
{ }_{*} \sum a_{n k}^{*} \odot z_{k} \longrightarrow \xi_{*} \odot \xi_{k}
$$

for every $z \in \omega^{*}$. Also, the sufficiency for the case $p \geq 1$ can be obtained in a similar way.

Conversely suppose that $(A z)_{n}$ exists for each $n \geq 1$ whenever $x \in \Omega$. Thus, the functionals

$$
\ddot{\|}_{*} \sum_{\nu} a_{n k}^{*} \odot z_{k} \ddot{\|} \in \Omega^{*},
$$

for each $n$ and each $v \geq 0$. They are $*$-linear and $*$-continuous since

$$
\ddot{\|} \sum_{v} a_{n k}^{*} \odot z_{k} \ddot{\|} \ddot{\leq} A_{v}^{1}(n) \ddot{\times} \ddot{2}^{(v / p)_{\beta}} \ddot{\times} \ddot{\|} z \ddot{\|}_{p}^{1 / p} .
$$

From Corollary 22, it follows that $\lim _{v *} \sum_{v} a_{n k}^{*} \odot z_{k}=(A z)_{n}$ is in $\Omega^{*}$, whence

$$
\ddot{\|}(A z)_{n} \ddot{\|} \ddot{\leq}\left\|A_{n}\right\| \ddot{x} \ddot{\|} z \ddot{\|}_{p}^{(1 / p)_{\beta}}
$$

where $\left\|A_{n}\right\|=\sup _{n} \ddot{\|}(A z)_{n} \ddot{\|} \ddot{\times} \ddot{\|} z \ddot{\|}_{p}^{(-1 / p)_{\beta}}$. Taking any integer $s>0$ and define $z \in \Omega$ by $z_{k}=0^{*}$ for $k \geq 2^{s+1}$ and $z_{N_{v}}=\ddot{2}^{(v / p)_{\beta}} \ddot{x} \operatorname{sgn} z_{n, N_{v}}, z_{k}=0^{*}\left(k \neq N_{v}\right)$, for $0 \leq v \leq s$, where $N_{v}$ is such that $\ddot{\|} z_{n, N_{v}} \ddot{\|}=\max _{v} \ddot{\|} a_{n, k} \ddot{\|}$. By (45) we have $\sum_{\beta}^{s} A_{\nu=0}^{1}(n) \ddot{\times} \ddot{2}^{(v / p)_{\beta}} \ddot{\leq}\left\|A_{n}\right\|$ for each $n$ and implies

$$
\sum_{\gamma=0}^{\infty} A_{\nu}^{1}(n) \ddot{\times} \ddot{2}^{(v / p)_{\beta}} \ddot{\leq}\left\|A_{n}\right\| \ddot{<} \infty .
$$

In fact the series $(A z)_{n}$ was absolutely $*$-convergent which gives

$$
\left\|A_{n}\right\| \ddot{\leq} \sum_{\gamma=0}^{\infty} A_{\nu}^{1}(n) \ddot{\times} \ddot{2}^{(v / p)_{\beta}} .
$$


From (46) and (47), it can easily be seen that

$$
\left\|A_{n}\right\|=\sum_{\gamma=0}^{\infty} A_{\nu}^{1}(n) \ddot{\times} \ddot{2}^{(v / p)_{\beta}} .
$$

This leads us together with Corollary 22 to the fact that

$$
\sup _{n}\left\|A_{n}\right\|=\sup _{n}\left\{\sum_{\gamma=0}^{\infty} \ddot{2}^{(v / p)_{\beta}} \ddot{\times} A_{\nu}^{1}(n)\right\} \ddot{<} \infty .
$$

Similarly, one can prove that (ii) ${ }^{\prime}$ is necessary condition. This completes the proof.

Now, as an immediate consequence of Theorem 23, the following corollary shows the $\beta$-duals for $\omega_{0}^{p}(\alpha, \beta), \omega^{p}(\alpha, \beta)$, and $\omega_{\infty}^{p}(\alpha, \beta)$.

Corollary 24. The $\beta$-duals of the spaces $\omega_{0}^{p}(\alpha, \beta), \omega^{p}(\alpha, \beta)$, and $\omega_{\infty}^{p}(\alpha, \beta)$ are $\mathscr{M}_{p}^{(\alpha, \beta)}$.

Remark 25. The symbol “ $\beta$ ” used for $\beta$-dual has different meaning from that of $\beta$ generator used in the text.

We may give with quoting the following proposition and lemma without proofs (see $[21,22]$ ) which are needed in the proof of next theorem.

Proposition 26. Let $X$ be $a * B K$-space. Then we have $A \in$ $\left(X: \ell_{\infty}^{*}\right)$ if and only $A_{n} \in X^{\beta}$ for all $n \in \mathbb{N}$ where $A_{n}=\left(a_{n k}^{*}\right)$ for the sequence in the $n$th row of $A$ for all $k \in \mathbb{N}$.

Lemma 27. Let $A=\left(a_{n k}^{*}\right)$ be an innite matrix. If $\left\|A_{n}\right\|_{\mathscr{M}_{p}^{(\alpha, \beta)}} \ddot{<} \infty$ and ${ }^{\beta} \lim _{n \rightarrow \infty}\left\|A_{n}\right\|_{\mathscr{M}_{p}^{(\alpha, \beta)}}=\ddot{0}$, then $\left\|A_{n}\right\|_{\mathscr{M}_{p}^{(\alpha, \beta)}}$ uniformly $*$-converges in $n \in \mathbb{N}$.

Theorem 28. The following statements hold:

(i) $A=\left(a_{n k}^{*}\right) \in\left(\omega_{\infty}^{p}(\alpha, \beta): \ell_{\infty}^{*}\right)=\left(\omega_{0}^{p}(\alpha, \beta): \ell_{\infty}^{*}\right)=$ $\left(\omega^{p}(\alpha, \beta): \ell_{\infty}^{*}\right)$ if and only if

$$
\sup _{n \in \mathbb{N}}\left\|A_{n}\right\|_{M_{p}^{(\alpha, \beta)}} \ddot{<} \infty \text {. }
$$

(ii) $A=\left(a_{n k}^{*}\right) \in\left(\omega_{\infty}^{p}(\alpha, \beta): c_{0}^{*}\right)$ if and only if

$$
\beta \lim _{n \rightarrow \infty}\left\|A_{n}\right\|_{\mathscr{M}_{p}^{(\alpha, \beta)}}=\ddot{0} \text {. }
$$

(iii) $A=\left(a_{n k}^{*}\right) \in\left(\omega_{0}^{p}(\alpha, \beta): c_{0}^{*}\right)$ if and only if (50) holds and

$$
{ }^{*} \lim _{n \rightarrow \infty} a_{n k}^{*}=0^{*}
$$

for all $k \in \mathbb{N}$.

(iv) $A=\left(a_{n k}^{*}\right) \in\left(\omega^{p}(\alpha, \beta): c_{0}^{*}\right)$ if and only if (50), (52) hold and

$$
{ }^{*} \lim _{n \rightarrow \infty} \sum_{k=1}^{\infty} a_{n k}^{*}=0^{*}
$$

Proof. (i) Condition (50) for $A \in\left(\omega_{0}^{p}(\alpha, \beta): \ell_{\infty}^{*}\right)$ follows from Proposition 26 and Corollary 24. Then the other parts follows from the fact that $\omega_{0}^{p}(\alpha, \beta) \subset \omega^{p}(\alpha, \beta) \subset \omega_{\infty}^{p}(\alpha, \beta)$ by Proposition 20.

(ii) This condition is proved in the same way as in Theorem 23 with $\xi_{k}=0 *$ for all $k \in \mathbb{N}$.

(iii) Since $\omega_{0}^{p}(\alpha, \beta)$ is a $* \mathrm{BK}$-space with $* \mathrm{AK}$ by Proposition 20 and $c^{*}, c_{0}^{*}$ are closed subset of $\ell_{\infty}^{*}$ the conditions follow from the characterization of $\left(\omega_{0}^{p}(\alpha, \beta): \ell_{\infty}^{*}\right)$.

(iv) The conditions follow from those in (iii).

\section{Concluding Remarks}

Non-Newtonian calculus is a methodology that allows one to have a different look at problems which can be investigated via calculus. It should be clear that the non-Newtonian calculus is a self-contained system independent of any other system of calculus. Therefore the reader may be surprised to learn that there is a uniform relationship between the corresponding operators of this calculus and the classical calculus.

In this paper we have introduced the sequence spaces $\omega_{0}^{p}(\alpha, \beta), \omega^{p}(\alpha, \beta)$, and $\omega_{\infty}^{p}(\alpha, \beta)$ as a generalization of the spaces $\omega_{0}^{p}, \omega^{p}$, and $\omega_{\infty}^{p}$ of Maddox [19]. Our main purpose is to determine the $\beta$-duals of the new spaces $\omega_{0}^{p}(\alpha, \beta), \omega^{p}(\alpha, \beta)$, and $\omega_{\infty}^{p}(\alpha, \beta)$ and is to characterize the classes of matrix transformations from these spaces to any one of the spaces $\ell_{\infty}^{*}, c^{*}$, and $c_{0}^{*}$. As a future work we will try to obtain the characterizations of the classes of infinite matrices from the spaces $\omega_{0}^{p}(\alpha, \beta), \omega^{p}(\alpha, \beta)$, and $\omega_{\infty}^{p}(\alpha, \beta)$ to a sequence space $\lambda$ over the non-Newtonian complex field different from $\ell_{\infty}^{*}$, $c^{*}$, and $c_{0}^{*}$.

\section{Competing Interests}

The author declares that there are no competing interests.

\section{References}

[1] M. Zeltser, M. Mursaleen, and S. A. Mohiuddine, "On almost conservative matrix methods for double sequence spaces," Publicationes Mathematicae, vol. 75, no. 3-4, pp. 387-399, 2009.

[2] U. Kadak and P. Baliarsingh, "On certain Euler difference sequence spaces of fractional order and related dual properties," The Journal of Nonlinear Science and Applications, vol. 8, no. 6, pp. 997-1004, 2015.

[3] E. Malkowsky, M. Mursaleen, and S. Suantai, "The dual spaces of sets of difference sequences of order $m$ and matrix transformations," Acta Mathematica Sinica, vol. 23, no. 3, pp. 521-532, 2007.

[4] E. Malkowsky and M. Mursaleen, "Matrix transformations between FK-Spaces and the sequence spaces $m(\varphi)$ and $n(\varphi)$," Journal of Mathematical Analysis and Applications, vol. 196, no. 2, pp. 659-665, 1995.

[5] M. Stieglitz and H. Tietz, "Matrixtransformationen von Folgenräumen Eine Ergebnisübersicht," Mathematische Zeitschrift, vol. 154, no. 1, pp. 1-16, 1977. 
[6] F. Başar, Summability Theory and Its Applications, Normed and Paranormed Sequence Spaces, Bentham Science Publishers, Istanbul, Turkey, 2012.

[7] M. Grossman, Bigeometric Calculus, Archimedes Foundation Box 240, Rockport, Mass, USA, 1983.

[8] M. Grossman and R. Katz, Non-Newtonian Calculus, Newton Institute, 1978.

[9] M. Grossman, The First Nonlinear System of Differential and Integral Calculus, Mathco, Rockport, Mass, USA, 1979.

[10] A. E. Bashirov, E. M. Kurpınar, and A. Özyapıc1, "Multiplicative calculus and its applications," Journal of Mathematical Analysis and Applications, vol. 337, no. 1, pp. 36-48, 2008.

[11] D. Aniszewska, "Multiplicative Runge Kutta methods," Nonlinear Dynamics, vol. 50, no. 1-2, pp. 265-272, 2007.

[12] E. Misirli and Y. Gurefe, "Multiplicative Adams-BashforthMoulton methods," Numerical Algorithms, vol. 57, no. 4, pp. 425-439, 2011.

[13] A. F. Çakmak and F. Başar, "Some new results on sequence spaces with respect to non-Newtonian calculus," Journal of Inequalities and Applications, vol. 2012, article 228, 2012.

[14] A. F. Çakmak and F. Başar, "Certain spaces of functions over the field of non-Newtonian complex numbers," Abstract and Applied Analysis, vol. 2014, Article ID 236124, 12 pages, 2014.

[15] S. Tekin and F. Başar, "Certain sequence spaces over the nonNewtonian complex field," Abstract and Applied Analysis, vol. 2013, Article ID 739319, 11 pages, 2013.

[16] U. Kadak, "Determination of the Köthe-Toeplitz duals over the non-Newtonian complex field," The Scientific World Journal, vol. 2014, Article ID 438924, 10 pages, 2014.

[17] U. Kadak and H. Efe, "The construction of Hilbert spaces over the non-Newtonian field," International Journal of Analysis, vol. 2014, Article ID 746059, 10 pages, 2014.

[18] U. Kadak and M. Özlük, "Generalized Runge-Kutta method with respect to the non-Newtonian calculus," Abstract and Applied Analysis, vol. 2015, Article ID 594685, 10 pages, 2015.

[19] I. J. Maddox, “On Kuttner's theorem," Journal of the London Mathematical Society, vol. 43, pp. 285-290, 1968.

[20] E. Malkowsky and V. Rakocevic, "An introduction into the theory of sequence spaces and measure of noncompactness," Zbornik Radova, vol. 9, no. 17, pp. 143-274, 2000.

[21] A. M. Jarrah and E. Malkowsky, "Ordinary, absolute and strong summability and matrix transformations," Filomat, no. 17, pp. 59-78, 2003.

[22] F. Başar, E. Malkowsky, and B. Altay, "Matrix transformations on the matrix domains of triangles in the spaces of strongly C1summable and bounded sequences," Publicationes Mathematicae, vol. 73, no. 1-2, pp. 193-213, 2008. 


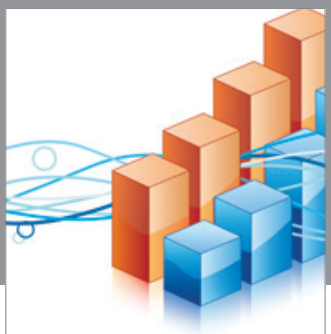

Advances in

Operations Research

vatem alat4

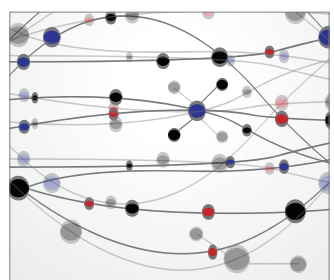

\section{The Scientific} World Journal
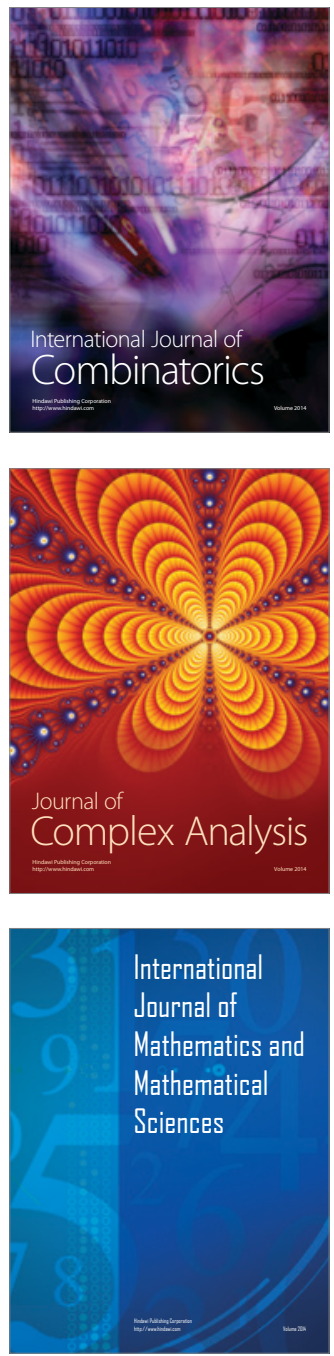
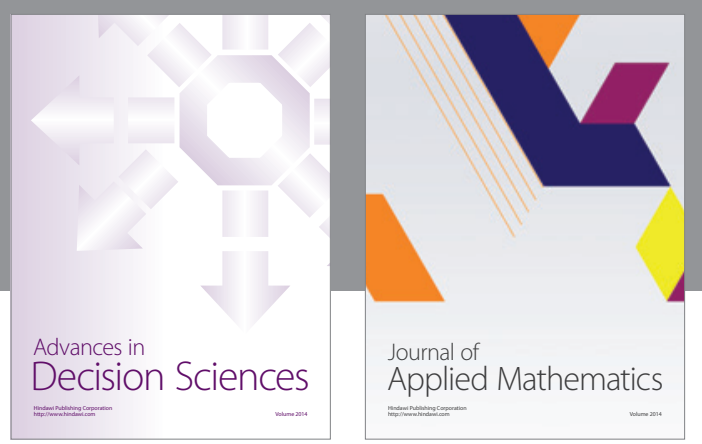

Algebra

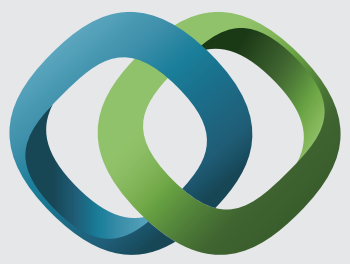

\section{Hindawi}

Submit your manuscripts at

http://www.hindawi.com
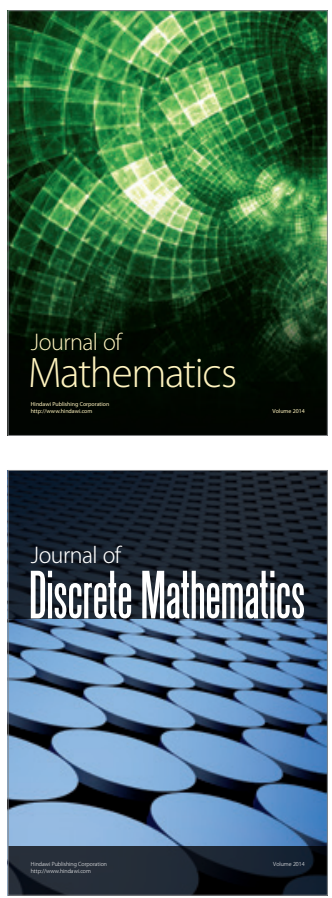

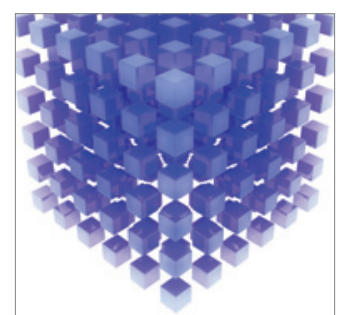

Mathematical Problems in Engineering
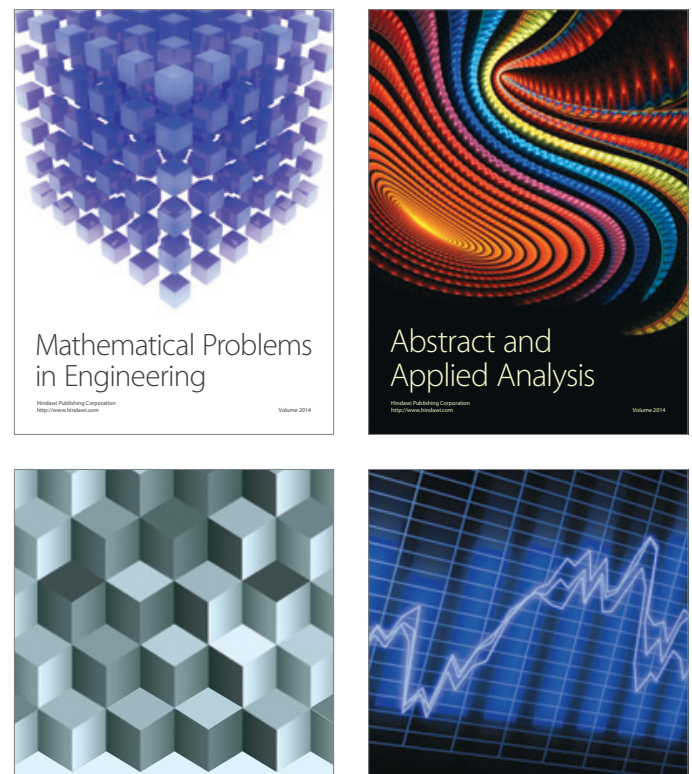

Journal of

Function Spaces

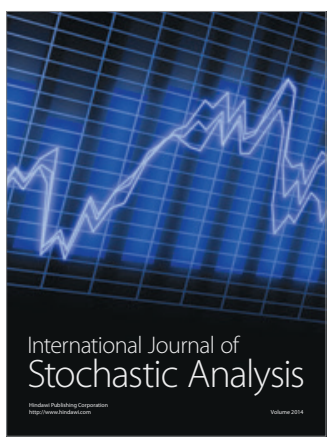

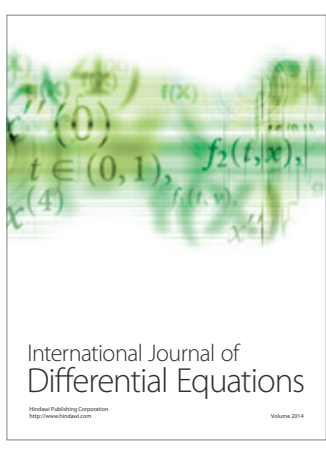
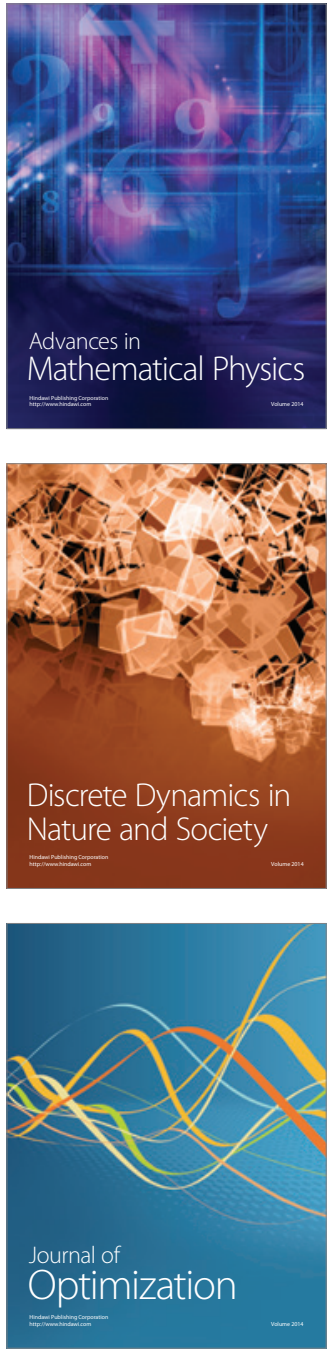